\title{
Assessment of size ordered recruitment
}

\author{
Parveen N. S. Bawa ${ }^{1}$, Kelvin E. Jones ${ }^{2 *}$ and Richard B. Stein ${ }^{3}$ \\ ${ }^{1}$ Department of Biomedical Physiology and Kinesiology, Simon Fraser University, Burnaby, BC, Canada \\ 2 Faculty of Physical Education and Recreation, University of Alberta, Edmonton, AB, Canada \\ ${ }^{3}$ Department of Physiology, University of Alberta, Edmonton, AB, Canada \\ *Correspondence: kejones@ualberta.ca
}

Edited by:

Maria Piotrkiewicz, Polish Academy of Sciences, Poland

Reviewed by:

Piotr Krutki, University School of Physical Education in Poznan, Poland

Keywords: motor unit recruitment, human experimentation, size principle, muscle contraction, movement

The spinal motoneurons innervating a limb muscle are heterogeneous: they vary in diameter of cell bodies, axons and surface area of the dendritic trees, electrophysiological differences (e.g., input resistance, afterhyperpolarization, spike threshold) and contractile properties of the associated muscle units. Considering the range of motoneuron and corresponding muscle unit properties, the obvious question arose about how and which motoneurons were selected by the central nervous system for various types of contractions and movements. Elwood Henneman and his coworkers proposed an unequivocal simple pattern in a series of papers starting in 1957 from experiments conducted on reduced cat preparations (Henneman, 1957; Henneman et al., 1974). The Size Principle of motoneuron recruitment stated that for any net excitatory input to the motoneuron pool, motoneurons were recruited in an orderly fashion, always from small to large. Stein and coworkers demonstrated that the size principle generalized to voluntary isometric contractions in humans, which they called Orderly Recruitment (Milner-Brown et al., 1973). The literature on this topic is well summarized in a recent review by Heckman and Enoka (2012).

Since the publication of Henneman's seminal paper in 1957, publications on this topic have steadily accumulated. The purpose of this short article is to clarify the basic essence of size ranked or orderly recruitment of motoneurons by addressing conclusions about the alternative: selective recruitment. This requires stretching the strict boundaries imposed by some authors in understanding the Size
Principle. For example, the anatomically defined motoneuron pools may not always coincide with the group of motoneurons being excited for a particular task; the precision of rank-ordered recruitment suggested from reduced animal preparations (Henneman et al., 1974) may not be that precise in a noisy physiological system (Stein et al., 2005). Here we elaborate on some of the factors that may have lead to the conclusions of selective or random recruitment.

Though the size principle was stated for a motoneuron pool, the initial observations made by Henneman and coworkers were not restricted to one pool. They recorded from ventral roots while stretching all triceps surae muscles; the recorded activity in individual ventral roots could have its origin in any one of the synergistic pools. One recorded action potential could be from soleus and the second from gastrocnemius. Yet orderly recruitment was observed between the collection of all motoneurons that were excited by the input, specifically the total motoneuron population of soleus, medial and lateral gastrocnemius (LG) muscles. Likewise, an anatomically defined motoneuron pool may under certain conditions be compartmentalized into task groups (Riek and Bawa, 1992) and orderly recruitment is observed within each task group. The existence of task groups does not imply size independent selective recruitment of motor unit types. Wakeling (2009) has reported recruitment of different compartments of triceps muscles in goats and humans depending on the mechanics of the movement. Selective recruitment of different compartments is akin to recruitment of different task groups; again, this observation does not imply selective recruitment if excitatory input is restricted to a subset of motoneurons. This same line of reasoning applies to the condition of fast paw shake in the cat (Smith et al., 1980). In these experiments EMG activity was observed in the fast LG muscle but not in the slow soleus muscle during the paw shake. This observation is often cited as evidence for selective recruitment of motor unit types. However, there was no discussion as to whether the motor units within the LG muscle, which is composed of type I and II muscle fibers, were recruited according to size. A subsequent study that included EMG sampling from histochemically regionalized muscles showed high activation of all muscle regions during paw shaking, which is not consistent with preferential recruitment of muscle regions rich in type II fibers during this condition (Chanaud et al., 1991). We suggest that when discussing recruitment order, the motoneuron pool should be operationally defined as the group of motoneurons that receive excitatory synaptic input to drive the functional movement, not the pool of motoneurons defined by anatomy. The validity of the Size Principle should then be evaluated within this operationally defined motoneuron pool to determine if recruitment proceeds from small to large.

What do orderly recruitment and selective recruitment really mean? The accepted narrative is that orderly recruitment of motor units from small to large twitch force, results in a more precise control of force and movement; this precision is 
more important for small and mid range forces. By maintaining the same order of recruitment, the central nervous system minimizes the computational load across a wide range of desired outputs (Henneman et al., 1974). A range of quantitative theoretical studies support this qualitative description. For example, Senn et al. (1997) used an information theoretical approach to demonstrate that orderly recruitment maximizes information content of motoneuron output that in turn minimizes the error in muscle force generation. Selective recruitment, on the other hand, refers to the hypothesis that under certain conditions the central nervous system selects motor units to enhance the force and rate of force output irrespective of the rank order of the motor unit within a motoneuron pool. To achieve this goal, selective recruitment may use preferential inhibition of small motor units. The most commonly proposed examples of selective recruitment include ballistic contractions, lengthening contractions and the preferential recruitment of fast motor units during cutaneous stimulation. However, empirical evidence from a number of laboratories failed to support the hypothesis of selective recruitment in these conditions (reviewed by Heckman and Enoka, 2012). Electrical stimulation of some pathways could produce inhibition of small motor units and excitation of larger motor units. Yet none of the behavioral studies demonstrated selective recruitment of large units with inhibition of the small ones. A possible basis for this discrepancy comes from Kernell and Hultborn (1990). They proposed that the selective excitatory and inhibitory synaptic inputs change the gain of the inputoutput curve of the motoneuron pool. To increase the recruitment gain, the small motoneurons are biased with inhibitory currents. Alternatively, or concurrently, the large motoneurons can be biased with excitatory currents. The opposite synaptic bias scheme can be used to decrease the gain. All motoneurons receiving excitatory input to drive the final movement, despite underlying bias inputs, should be considered part of a functionally defined pool of motoneurons. In the high gain situation, there will be a higher likelihood of random departures from strict recruitment as a result of noise-however, the general principle of rank ordered recruitment will remain.

Another factor that has contributed to a misunderstanding of the orderly recruitment is the expectation for precise rank ordered recruitment (Henneman et al., 1974). The degree of precision suggested from reduced animal preparations is not expected to hold for a noisy physiological system (Stein et al., 2005). It is important to remember that rank-ordered recruitment was originally defined for movement under healthy conditions. However, in conditions such as ageing, reinnervation, pain and fatigue, when precision decreases, other processes may obscure rank-ordered recruitment. With ageing, some motoneurons die leaving behind orphaned muscle fibers. The surviving motoneurons will sprout new terminals to reinnervate some of the orphaned fibers, thus changing the size of the muscle units (Chan et al., 2001; Gordon et al., 2004). Under these conditions, the order of recruitment will be less orderly which will lead to a decline in precision of motor output. During prolonged contractions in healthy adults, it has been shown that motoneurons fatigue and rotation of activity occurs among motor units (Manning et al., 2010). If rotation occurs among almost similar sized motor units, precision will remain unaffected. However, rotation among motor units of quite different sizes would increase the noise in motor output. Another example of disrupted rank ordered recruitment is the activity of motor units during pain (Tucker et al., 2009). These examples are not evidence for selective recruitment, but rather a decrease in precision of rank-ordered recruitment. In our experience the situation in which common sense reasoning favors selective recruitment is movements with maximum velocity over a short period of time: a ballistic movement. The reasoning is that slow motor units impede ballistic movements while exclusive recruitment of fast units would be optimal. However a recent study demonstrated that the recruitment of slow motor units does not pose any such problem (Holt et al., 2014).

The ultimate meaning of recruitment order lies in the force output of the entire musculoskeletal system for the purpose of behaviorally relevant movements. Clearly in laboratory conditions with simple muscles and a limited set of contractions, recruitment is orderly in terms of force (Milner-Brown et al., 1973; Calancie and Bawa, 1985; Zajac and Faden, 1985; Riek and Bawa, 1992; Jones et al., 1993). In biomechanically complex musculotendon-skeletal systems, accurately measuring the size of a motor unit may be difficult (Clamann and Schelhorn, 1988; Bodine-Fowler et al., 1990; Roy et al., 1995). The challenge for the field is to measure size and recruitment order, over relevant ranges of force during ecologically valid behavior (Jones et al., 1994). Until this is done, the question remains whether the laboratory defined size principle of orderly recruitment will generalize to movements of everyday life.

\section{ACKNOWLEDGMENT}

Kelvin E. Jones acknowledges grant support from NSERC, CIHR, and the ALS Bernice Ramsay grant.

\section{REFERENCES}

Bodine-Fowler, S., Garfinkel, A., Roy, R. R., and Edgerton, V. R. (1990). Spatial distribution of muscle fibers within the territory of a motor unit. Muscle Nerve 13, 1133-1145. doi: 10.1002/mus. 880131208

Calancie, B., and Bawa, P. (1985). Voluntary and reflexive recruitment of flexor carpi radialis motor units in humans. J. Neurophysiol. 53, 1194-1200.

Chan, K. M., Doherty, T. J., and Brown, W. F. (2001). Contractile properties of human motor units in health, aging, and disease. Muscle Nerve 24, 1113-1133. doi: 10.1002/mus.1123

Chanaud, C. M., Pratt, C. A., and Loeb, G. E. (1991). Functionally complex muscles of the cat hindlimb. V. The roles of histochemical fiber-type regionalization and mechanical heterogeneity in differential muscle activation. Exp. Brain Res. 85, 300-315. doi: 10.1007/BF00229408

Clamann, H. P., and Schelhorn, T. B. (1988). Nonlinear force addition of newly recruited motor units in the cat hindlimb. Muscle Nerve 11, 1079-1089. doi: 10.1002/mus. 880111012

Gordon, T., Thomas, C. K., Munson, J. B., and Stein, R. B. (2004). The resilience of the size principle in the organization of motor unit properties in normal and reinnervated adult skeletal muscles. Can. J. Physiol. Pharmacol. 82, 645-661. doi: 10.1139/y04-081

Heckman, C. J., and Enoka, R. M. (2012). Motor unit. Compr. Physiol. 2, 2629-2682. doi: 10.1002/cphy. c100087

Henneman, E. (1957). Relation between size of neurons and their susceptibility to discharge. Science 126, 1345-1347. doi: 10.1126/science.126. 3287.1345 
Henneman, E., Clamann, H. P., Gillies, J. D., and Skinner, R. D. (1974). Rank order of motoneurons within a pool: law of combination. J. Neurophysiol. 37, 1338-1349.

Holt, N. C., Wakeling, J. M., and Biewener, A. A. (2014). The effect of fast and slow motor unit activation on whole-muscle mechanical performace: the size principle may not pose a mechanical paradox. Proc. R. Soc. B Biol. Sci. 281:20140002. doi: 10.1098/rspb.2014.0002

Jones, K. E., Bawa, P., and McMillan, A. S. (1993). Recruitment of motor units in human flexor carpi ulnaris. Brain Res. 602, 354-356. doi: 10.1016/0006-8993(93)90702-O

Jones, K. E., Lyons, M., Bawa, P., and Lemon, R. N. (1994). Recruitment of motoneurons during various behavioural tasks. Exp. Brain Res. 100, 503-508. doi: 10.1007/BF02738409

Kernell, D., and Hultborn, H. (1990). Synaptic effects on recruitment gain: a mechanism of importance for the input-output relations of motoneuron pools? Brain Res. 507, 176-179. doi: 10.1016/00068993(90)90542-J

Manning, C. D., Miller, T. A., Burnham, M. L., Murnaghan, C. D., Calancie, B., and Bawa, P. (2010). Recovery of human motoneurons during rotation. Exp. Brain Res. 204, 139-144. doi: 10.1007/s00221-010-2295-2

Milner-Brown, H. S., Stein, R. B., and Yemm, R. (1973). The orderly recruitment oh human motor units during voluntary isometric contractions. J. Physiol. 230, 359-370.

Riek, S., and Bawa, P. (1992). Recruitment of motor units in human forearm extensors. J. Neurophysiol. 68, 100-108.

Roy, R. R., Garfinkel, A., Ounjian, M., Payne, J., Hirahara, A., Hsu, E., et al. (1995). Three-dimensional structure of cat tibialis anterior motor units. Muscle Nerve 18, 1187-1195. doi: 10.1002/mus.880181015

Senn, W. K., Wyler, K., Clamann, H. P., Kleinle, J., Lüscher, H.-R., and Müller, L. (1997). Size principle and information theory. Biol. Cybern. 76, 11-22. doi: 10.1007/s004220 050317

Smith, J. L., Betts, B., Edgerton, V. R., and Zernicke, R. F. (1980). Rapid ankle extensions during paw shakes: selective recruitment of fast ankle extensors. J. Neurophysiol. 43, 612-620.

Stein, R. B., Gossen, E. R., and Jones, K. E. (2005). Neuronal variability: noise or part of the signal? Nat. Rev. Neurosci. 6, 389-397. doi: 10.1038/ nrn1668

Tucker, K., Butler, J., Graven-Nielsen, T., Riek, S., and Hodges, P. (2009). Motor unit recruitment strategies are altered during deep-tissue pain. J. Neurosci. 29, 10820-10826. doi: 10.1523/JNEUROSCI.521108.2009

Wakeling, J. M. (2009). The recruitment of different compartments within a muscle depends on the mechanics of the movement. Biol. Lett. 5, 30-34. doi: 10.1098/rsbl.2008.0459

Zajac, F. E., and Faden, J. S. (1985). Relationship among recruitment order, axonal conduction velocity, and muscle-unit properties of typeidentified motor units in cat plantaris muscle. J. Neurophysiol. 53, 1303-1322.

Conflict of Interest Statement: The authors declare that the research was conducted in the absence of any commercial or financial relationships that could be construed as a potential conflict of interest.

Received: 09 June 2014; accepted: 30 June 2014; published online: 28 July 2014.

Citation: Bawa PNS, Jones KE and Stein RB (2014) Assessment of size ordered recruitment. Front. Hum. Neurosci. 8:532. doi: 10.3389/fnhum.2014.00532

This article was submitted to the journal Frontiers in Human Neuroscience.

Copyright (c) 2014 Bawa, Jones and Stein. This is an open-access article distributed under the terms of the Creative Commons Attribution License (CC BY). The use, distribution or reproduction in other forums is permitted, provided the original author(s) or licensor are credited and that the original publication in this journal is cited, in accordance with accepted academic practice. No use, distribution or reproduction is permitted which does not comply with these terms. 